\title{
BMJ Open Cardiac arrest as a reportable condition: a cohort study of the first 6 years of the Norwegian out-of-hospital cardiac arrest registry
}

Ingvild Beathe Myrhaugen Tjelmeland (D) ,,2 Kristin Alm-Kruse, ${ }^{3,4}$ Lars-Jøran Andersson, ${ }^{5}$ Ståle Bratland, ${ }^{5}$ Arne-Ketil Hafstad, ${ }^{6}$ Bjørn Haug, ${ }^{7}$ Jørund Langørgen, ${ }^{8}$ Alf Inge Larsen, ${ }^{9,10}$ Thomas Werner Lindner, ${ }^{11}$ Jan Erik Nilsen, ${ }^{1}$ Theresa M Olasveengen, ${ }^{4,12}$ Eldar Soreide, ${ }^{13,14}$ Eirik Skogvoll, ${ }^{15,16}$ Jo Kramer-Johansen ${ }^{1,4}$

To cite: Tjelmeland IBM, AlmKruse K, Andersson L-J, et al. Cardiac arrest as a reportable condition: a cohort study of the first 6 years of the Norwegian out-of-hospital cardiac arrest registry. BMJ Open 2020;10:e038133. doi:10.1136/ bmjopen-2020-038133

- Prepublication history and additional material for this paper are available online. To view these files, please visit the journal online (http://dx.doi. org/10.1136/bmjopen-2020038133).

Received 28 February 2020

Revised 23 April 2020

Accepted 12 May 2020
Check for updates

(c) Author(s) (or their employer(s)) 2020. Re-use permitted under CC BY-NC. No commercial re-use. See rights and permissions. Published by BMJ.

For numbered affiliations see end of article.

Correspondence to Ingvild Beathe Myrhaugen Tjelmeland; ingvild@nakos.no

\section{ABSTRACT}

Objectives The Norwegian Cardiac Arrest Registry (NorCAR) was established in 2013 when cardiac arrest became a mandatory reportable condition. The aim of this cohort study is to describe how the world's first mandatory, population-based cardiac arrest registry evolved during its first 6 years.

Setting Norway has a total population of 5.3 million inhabitants with a population density that varies considerably. All residents are assigned a unique identifier number, giving nationally approved registries access to information about all births and deaths in the country. Data in the registry are entered by data processors; public employees with close links to the emergency medical services. All data processors undergo a standardised training and meet for yearly retraining and updates.

Participants All events of cardiac arrest where bystanders or healthcare professionals have started cardiopulmonary resuscitation or performed defibrillation are included into the NorCAR.

Primary and secondary outcome measures Since the establishment of the registry, the number of reporting health trusts, the number of reported events and the corresponding population at risk were followed year by year. Outcome is measured as changes in inclusion rate, incidence per 100000 inhabitants and survival to 30 days after cardiac arrest.

Results In total, 14849 cases were registered over 6 years, between 2013 and 2018 . The number of health trusts reporting rose steadily from 2013. Within 3 years, all trusts reported to the registry with an increasing number of events reported; going from 1101 to 3400 per year. The prevalence of bystander cardiopulmonary resuscitation increased slightly, but the population incidence of survival did not change.

Conclusion Declaring cardiac arrest as a reportable condition and close follow-up of all reporting areas is essential when building a national registry.

\section{INTRODUCTION AND BACKGROUND}

Response, treatment and outcome after out-of-hospital cardiac arrest (OHCA) is an
Strengths and limitations of this study

- Cohort study of the world's first mandatory cardiac arrest registry presenting establishment and the first results.

- Includes almost 15000 cases of cardiac arrest.

- Merging information from a lot of different ambulance services uncovers challenges related to the understanding of the different data definitions.

- The Norwegian registry uses the national unique personal identifier number, and generalisability of the method might be limited.

internationally recognised quality indicator for the emergency medical service (EMS). ${ }^{1}$ The links in the chain-of-survival highlight both the time-sensitive nature of interventions and the concept that high quality in all stages of treatment is necessary to improve patient outcome. ${ }^{2}$

A population-based cardiac arrest registry allows systematic assessment of the various components and aspects of resuscitation care; early recognition of cardiac arrest, bystander cardiopulmonary resuscitation (CPR), handling of emergency calls, dispatch assisted CPR (DA-CPR), ambulance dispatch and care, and hospital treatment. Registry data may facilitate an understanding of how each element in a system affects survival and identify potential for improvement. ${ }^{3}$

The reported population incidence of OHCA in which CPR is initiated by a bystander or the EMS varies worldwide. A recent European study reports an incidence between 28 and 91 per 100000 inhabitants. The Australasian Resuscitation Outcomes Consortium (Aus-ROC) report similar estimates between 35 and 54 per 100000 inhabitants. ${ }^{5}$ The outcome after OHCA varies 
between and within countries. Sweden, establishing a voluntary registry already in 1990, reports the incidence of survival after OHCA between 3.7 and 10.4 per 100000 inhabitants, ${ }^{6}$ similar to prior publications from single centres in Norway. ${ }^{78}$

In 2013, Norwegian Cardiac Arrest Registry (NorCAR) became part of the Norwegian Cardiovascular Disease Registry (NCVDR) run by Norwegian Institute of Public Health and reporting to the registry became mandatory.

The aim of this paper is to describe how the inclusion rate, the incidence and estimated survival developed in this world's first mandatory, population-based cardiac arrest register over its first 6 years.

\section{METHODS \\ Setting}

Norway has 5.3 million inhabitants with a population density that varies considerably between urban and rural areas (Oslo 223 $/ \mathrm{km}^{2}$; Finnmark 1.6/ $\mathrm{km}^{2}$ ). The Norwegian single-payer public insurance covers all aspects of healthcare. Specialist healthcare is organised in 4 regional and 19 local Health Trusts. For medical emergencies, there is one dedicated medical emergency telephone number, 113, that terminates at 16 local Emergency Medical Communications Centres (EMCCs).

EMCCs are staffed with nurses and ambulance personnel, and dispatch the EMS. The EMS response may include ambulance, physician staffed helicopters/rapid response car, ambulance boats as well as first responders from the fire brigade and out-of-office-hours general practitioners, all dependent on the location of the arrest and the available resources. Telephone-guided first aid, including DA-CPR, has been an integrated EMCC routine since 1995. DA-CPR is provided to bystanders when the patient is unconscious and not breathing normally.

\section{Data management and governance}

All residents in Norway are assigned an 11-digit unique personal identifier number. This ID is a common identifier across all public registries, including health systems and can be used by approved national registries to confirm data about birth, name, address and date of death. Provisional ID can be generated for temporary residents or when ID is unknown.

Registration of diseases and procedures related to cardiovascular conditions in NCVDR is regulated to allow the collection, analysis and use of sensitive data. ${ }^{9}$ Reporting to NorCAR is mandatory as it is part of NCVDR, and there is no need for consent; rather all healthcare personnel and facilities have a duty to report. Through the online reporting system, the strictest level of patient privacy and data integrity is maintained in accordance with best practice and regulations.

Entry into NorCAR is made by data processors, often part-time nurses or paramedics with other tasks related to cardiac arrest education, organisation or quality improvement. All data processors are employed by the local Health Trusts. To ensure equal understanding of data definitions, data processors undergo standardised training and meet for yearly retraining and updates.

NorCAR is governed by a national advisory board representing all Regional Health Trusts, the Norwegian Resuscitation Council, the Norwegian Society of Cardiology, the Norwegian Myocardial Infarction Registry and the National Advisory Unit of Prehospital Emergency Medicine. The board also includes a data processor and a patient representative.

\section{Patient and public involvement}

The user representative involved in this project is part of NorCARs steering committee. He represents the patient organisation National Association of Heart and Lung Disease that has 54000 members across Norway suffering from cardiovascular diseases, including myocardial infarction, heart failure and stroke. He provides a channel for communication to the patient population as well as to the boards of health trusts through his network of fellow user representatives. He has been involved in the development of this research project.

\section{Event registration}

All patients that are unresponsive without normal breathing, where bystander, first responders, EMS or other healthcare professionals initiate CPR or defibrillation, are included in the registry. DA-CPR is defined as treatment alongside basic or advanced CPR or defibrillation. Patients are included irrespective of having a confirmed cardiac arrest on EMS arrival or not. Patients who do not receive any CPR are not included, neither are newborns in need of resuscitation at birth.

\section{Data sources}

Registration of an event often starts in the EMCG when DA-CPR is initiated. After the event the attending ambulance personnel fill in a dedicated form and transfer the information to the data processor. The local data processors collects information on the event and the patient, checks data quality and collect additional information from the defibrillator and hospital records when applicable. To ensure compete local coverage, data processors routinely check for missing cases in the EMCC databases, air-ambulance patient report forms, hospital electronic records and the Norwegian Patient Registry. Several logical checks have been implemented in the electronic report form, preventing typing errors or non-logical entries and finally the national registry staff performs a second quality control of all submitted data.

\section{Variables}

The dataset in NorCAR adheres to the Utstein dataset and definitions,${ }^{10}$ with some minor modifications to adjust for local conditions, and include information on the event, treatment and outcome. A full description of our dataset can be found in the online supplementary appendix. 


\section{Statistical methods}

When calculating bystander CPR rates, all EMS witnessed cardiac arrests are excluded from the analysis. When calculating the incidence of ROSC and 30-day survival, only patients treated by EMS, or patients who have been successfully resuscitated using an Automatic External Defibrillator (AED) before EMS arrival, are included.

Descriptive measures are provided as mean with SD or median with range, as appropriate according to the distribution of data. For regions reporting part of a year, we calculate population incidence by dividing number of patients by the corresponding fraction of the person-years for that region. Yearly trends in the number of reported events, the population incidence and the incidence of survivors with 95\% CIs were investigated using Poisson regression with year as predictor variable. Yearly trends in the prevalence of bystander CPR were investigated with logistic regression with year as predictor variable. A $\mathrm{p}<0.05$ was considered as statistically significant. The software $\mathrm{R}^{11}$ was used for the statistical analyses.

\section{Data protection}

NorCAR is a national person-identifiable health registry that does not require consent from the registered individual or next of kin. ${ }^{9}$

\section{RESULTS}

\section{Regional and national development since inception}

A total of 14849 OHCA events were registered in NorCAR from 2013 to 2018. The number of participating health trusts rose steadily, and by May 2016 data collection and reporting was established in all of Norway (figure 1).

The reported number of OHCA per year increased from 1101 in 2013 to 3400 in 2018 (figure $2, \mathrm{p}<0.01$ ). The estimated population incidence also increased from 46 (95\% CI 45 to 50) to 64 (62 to 66) per 100000 inhabitants $(p<0.01)$. The incidence of OHCAs treated by ambulance increased from 44 to 53 per 100000 inhabitants $(\mathrm{p}<0.01)$. (table 1)

\section{Utstein comparator group}

The Utstein comparator group of $2014^{10}$ is defined as patients experiencing witnessed cardiac arrest where the first documented rhythm is shockable. In addition, we

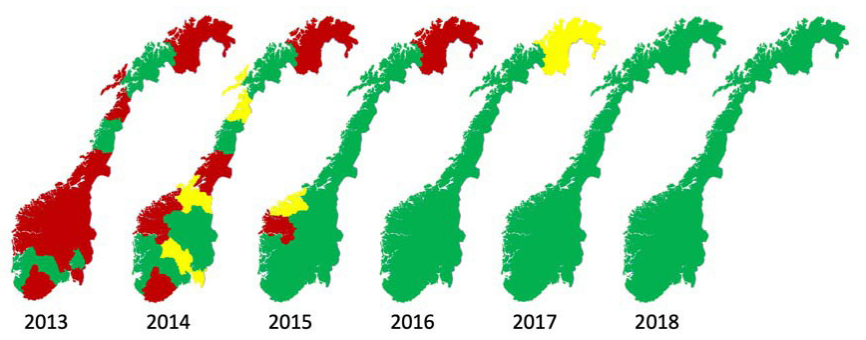

Figure 1 Map of Norway showing health trusts that report data to the National registry. Green: data collected the entire year, yellow: data collected for parts of the year, red: did not report.

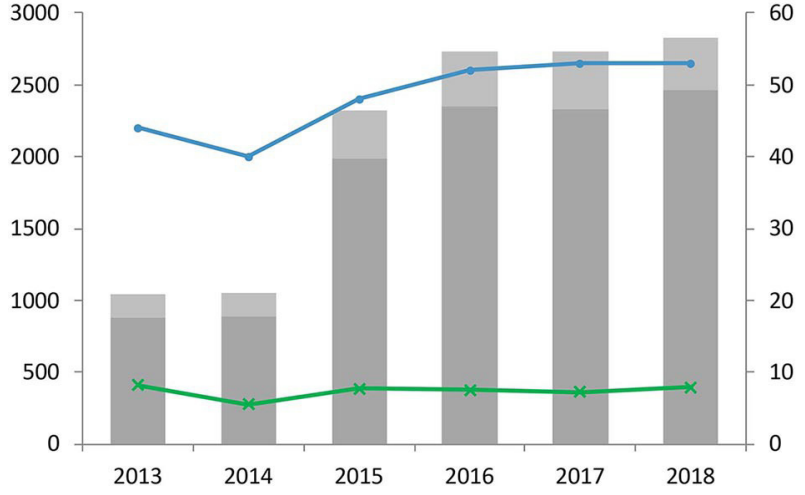

Figure 2 All patients included in the registry. Left axis and bars: number of out-of-hospital cardiac arrests treated by ambulance, by year of registration. Patients with EMSobserved collapse are identified as light grey. Right axis and lines: population incidence of ambulance treated arrests (blue), and population incidence of 30-day survival (green); per 100000 inhabitants. EMS, emergency medical service.

have included witnessed cardiac arrest-patients successfully resuscitated before EMS arrival to our Utstein comparator group. These patients have been defibrillated before EMS personnel arrive on scene and have spontaneous circulation when EMS arrives (shown as orange in figure 3 ).

The incidence of the Utstein comparator group has varied between 7.4 and 8.9 per 100000 inhabitants, with a survival incidence in this group of 3.5 per 100000 inhabitants $(42 \%)$. The incidence of ambulance witnessed cardiac arrests with shockable first rhythm has been about 2 per 100000 inhabitants with a survival incidence of 1.2 per 100000 inhabitants $(63 \%)$. The survivors in the Utstein comparator group and ambulance witnessed arrests constitute more than $70 \%$ of the total number of 30-day survivors (figure 3 ).

\section{Patient demography and outcome}

Median age was 65 (IQR 55-79) years and $67 \%$ were male. In $80 \%$ of the cases, CPR was started before EMS arrival (table 1). Among the 13003 ambulance treated patients, 15 per 100000 inhabitants (30\%) achieved return of spontaneous circulation (ROSC) that lasted long enough for hospital admission or for transferral to the next treating prehospital unit (sustained ROSC).

Likewise, 30-day survival was 7.1 per 100000 inhabitants $(14 \%)$ of the ambulance treated patients, and did not change over the period investigated $(\mathrm{p}=0.98)$. If we include patients defibrillated before EMS arrival, the overall survival is 7.4 per 100000 inhabitants (15\%).

\section{DISCUSSION}

The main findings from the first 6 years of the world's first mandatory cardiac arrest registry was an initial increase in the reported incidence of OHCA, levelling off after a few years. We observed a stable incidence of survival at 30 days as well as a bystander CPR rate of $80 \%$ during 


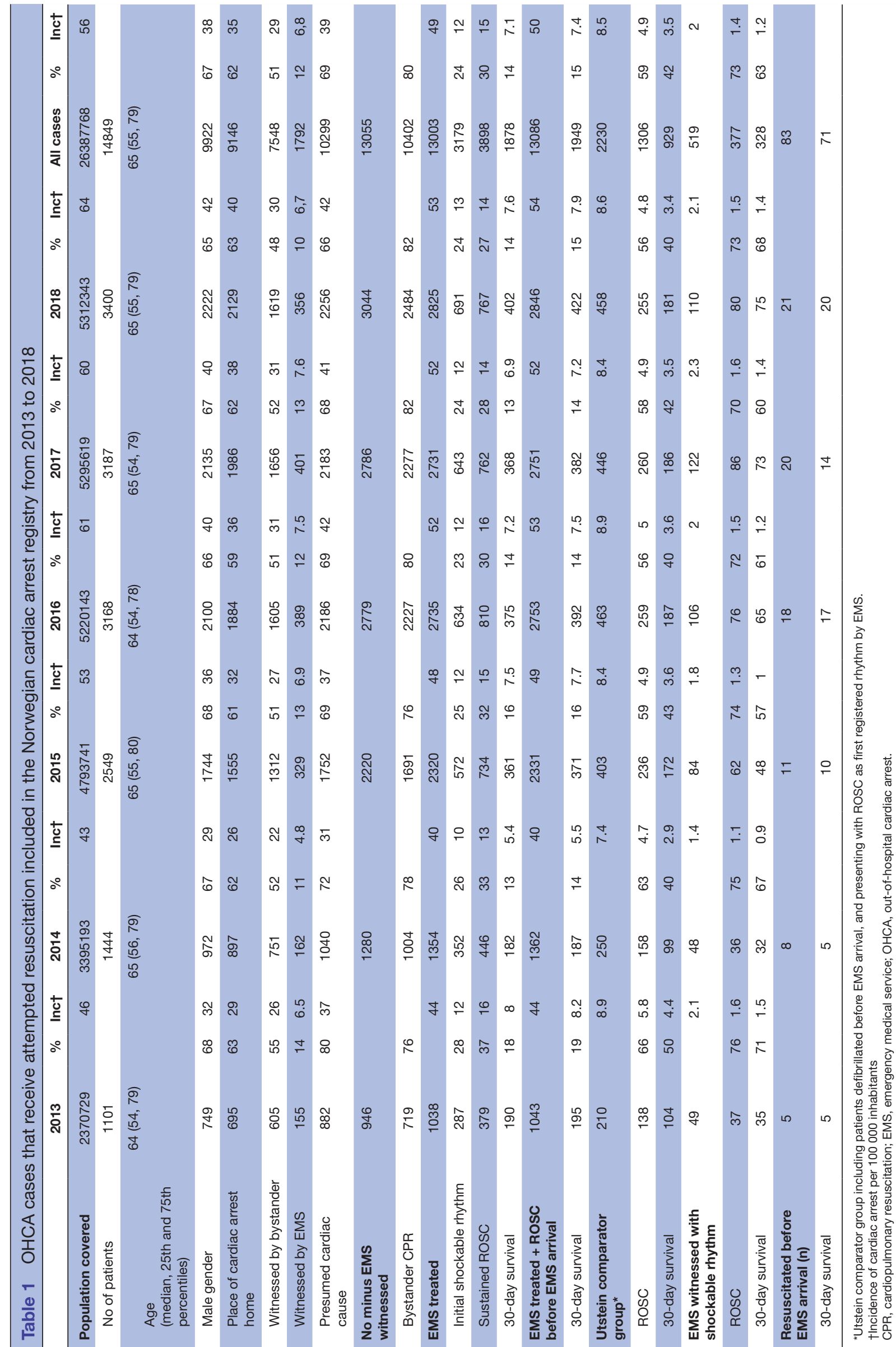




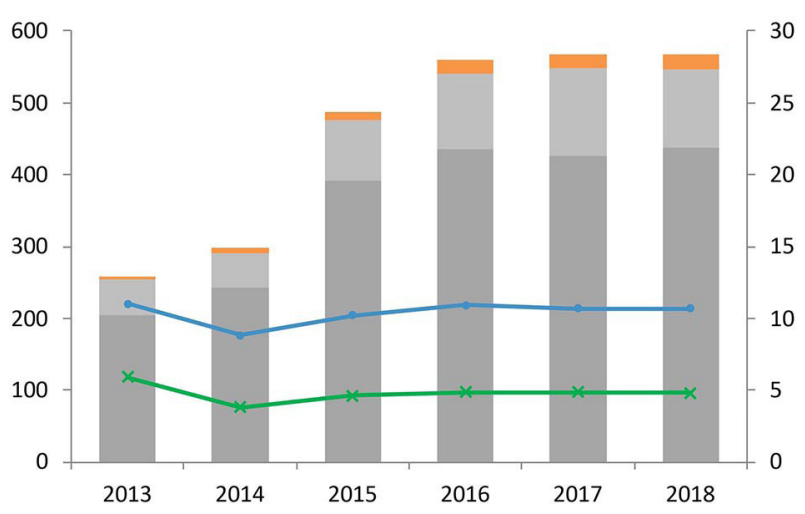

Figure 3 The Utstein comparator group (witnessed collapse and shockable first rhythm). Left axis and bars: number of cardiac arrest patients by year of registration. Patients with EMS-observed collapse are identified as light grey. Patients successfully resuscitated with AED before EMS arrival are orange. Right axis and lines: population incidence of the total Utstein comparator group (including EMS witnessed cases and those resuscitated before EMS arrival) (blue line), and population incidence of 30-day survival in the total group (green); per 100000 inhabitants. AED, Automatic External Defibrillator; EMS, emergency medical service.

the whole observation period. The likely explanation for the increasing number of events is a more complete registration.

The reported rate of bystander CPR of $80 \%$ is among the highest ever published. ${ }^{4}$ It is not fully understood why Norway has such high bystander CPR rate, but DA-CPR being implemented in all EMCCs already in 1995, a culture of willingness to help as well as repeated CPR training in school and adult life gives our inhabitants knowledge of and a willingness to perform CPR. The historical background also includes the first ever resuscitation training manikin being 'born' in Norway in $1960 .^{12}$ The current public strategy to increase bystander CPR involves collaborations between the Norwegian Health Directorate, nongovernmental organisations and EMCCs. ${ }^{13}$ A study of the term 'Bystander CPR' ${ }^{14}$ and an article from Aus-ROC ${ }^{15}$ both underline the importance of clear definitions of what constitutes a 'bystander'. In the Norwegian registry, we have not seen any big changes in the rate of bystander CPR over the years, indication that the interpretation has not changed, and that the high bystander rates are representative for our nation.

The initial increases seen in total numbers and ambulance treated cardiac arrests per population at risk, has levelled off. We believe that this is due to an initial underreporting of patients that were not treated by ambulance because they had clear signs of death, or the patient did not survive the event and was not transported to hospital. The stable survival rate at around 7 per 100000 inhabitants, despite an increase in overall reported incidence, supports this. Reports from Denmark ${ }^{16}$ and Sweden ${ }^{17}$ indicate that survivors might also be under-reported to national registries, and a validation process of inclusions would further strengthen the registry.
As the number of included patients increase, the proportion of survivors decrease, again stressing the importance of reporting results as incidence rates in addition to percentages. Consistent method for reporting the number of cardiac arrests, compared with the number of inhabitants in the region covered, makes it possible to compare results across regions and between countries as suggested by Becker et $a l^{18}$ Comparison of results can also be done for subgroups of patients, such as the Utstein Comparator group (witnessed and shockable first rhythm). As this group is more homogeneous in cause and treatment, percentages may be appropriate. In the Norwegian registry, ROSC rate and survival to 30 days in the Utstein comparator group were $59 \%$ and $42 \%$, respectively. The ROSC rate is similar to the overall ROSC rate for this group in the EuReCa-two study, but the survival to 30 days is higher than reported both in the European study and in the Aus-ROC epistry. ${ }^{45}$

Some patients do not receive EMS treatment though bystanders have started CPR before ambulance arrival (12\%, table 1). The reasons for EMS personnel not to start treatment can be signs of death, do-not-resuscitateorders, further resuscitation is considered futile due to circumstances or comorbidities, or that the patient presents a pulse and signs of life on EMS arrival. Patients who have received bystander CPR, but have a pulse when EMS arrives, cannot be considered confirmed cardiac arrests. To be consistent and conservative, we do not include these patients when calculating survival with one exception; the patient has received a shock from an AED before EMS arrival. The number of reported patients in this group has increased from 5 in 2013 to more than 20, adding up to 83 patients over 6 years. The increase in the reported numbers could be due to a better reporting combined with increasing availability of AEDs in public places, use of first responders and the implementation of an AEDregistry directly available in the maps in the EMCC. ${ }^{13}$ It is, however, important to acknowledge that there are a lot of barriers to an increased use of AED and one such barrier is that the majority of cardiac arrests occurring at home $(62 \%)$.

\section{Limitations of the study}

Collecting data from different local registries has resulted in some challenges related to the understanding of the different data definitions in the Utstein template. Translating the dataset and distributing it to all EMS personnel takes time, meaning that differences between Health Trusts the first years of the registry might be related to different interpretation of the definitions just as well as real differences. We hope that the continuous work from the registry, including standardised training and yearly meetings has contributed to a common interpretation. It is also unknown if the focus on treatment of OHCA has changed registration practices or if there is a changing culture. ${ }^{18}$

Missing information in the registry is handled by returning the registration to the relevant health trust, 
who then correct the data. Missing information that cannot be retrieved is handled by excluding the patient from the relevant analysis. It is, however, a challenge that by the time the national registry returns the form to the data processor, the cardiac arrest happened more than 3 months ago. Retrieving information more than 3 months after the event is difficult, and recall bias is substantial.

\section{CONCLUSION}

Having cardiac arrest as a reportable condition and closely following up all submitted registry data is essential when building a national registry. Defining cardiac arrest as a reportable condition made it possible to include all regions of Norway within a 4-year period, and increased the number of reported cardiac arrests cases to an incidence of 64 per 100000 inhabitants. Survival to 30 days stayed stable with an incidence of 7.4 per 100000 inhabitants.

\section{Author affiliations}

${ }^{1}$ Division of Prehospital Services, Oslo University Hospital, Oslo, Norway ${ }^{2}$ Institute for Emergency Medicine, University Hospital Schleswig Holstein, Kiel, Germany

${ }^{3}$ Division of Emergencies and Critical Care, Oslo University Hospital, Oslo, Norway

${ }^{4}$ Institute of Clinical Medicine, University of Oslo, Oslo, Norway

${ }^{5}$ Clinic of Emergency Medicine, University Hospital of North Norway, Tromso, Norway

${ }^{6}$ National Association for Heart and Lung Disease, Jessheim, Norway

${ }^{7}$ Department of Cardiology, Akershus University Hospital, Lorenskog, Norway

${ }^{8}$ Department of Heart Disease, Haukeland University Hospital, Bergen, Norway

${ }^{9}$ Department of Cardiology, Stavanger University Hospital, Stavanger, Norway

${ }^{10}$ Department of Clinical Science, University of Bergen, Bergen, Norway

${ }^{11}$ Regional Competency Center for Emergency Medicine (RAKOS), Stavanger

University Hospital, Stavanger, Norway

${ }^{12}$ Department of Research and Development and Department of Anesthesiology, Oslo University Hospital, Oslo, Norway

${ }^{13}$ Critical Care and Anaesthesiology Research Group, Stavanger University Hospital, Stavanger, Norway

${ }^{14}$ Department of Clinical Medicine, University of Bergen, Bergen, Hordaland, Norway

${ }^{15}$ Department of Circulation and Medical Imaging, Norwegian University of Science and Technology, Trondheim, Norway

${ }^{16} \mathrm{Clinic}$ of Anaesthesia and Intensive Care, St. Olav University Hospital, Trondheim, Norway

Acknowledgements The authors wish to thank all the local data processors and all former members of the steering committee; Petter Andreas Steen, Mads Gilbert, Kaare Harald Bønaa, Are Helseth and Per Halvorsen.

Contributor IBMT: contributed in the conception, planning, design, acquisition of data, analysis and interpretation of data, first draft of article, revision of draft and approval of final manuscript. KA-K: contributed in the design, acquisition of data, analysis and interpretation of data, revision of draft and approval of final manuscript. L-JA: contributed in the conception, planning, design, revision of draft and approval of final manuscript. SB: contributed in the conception, planning, revision of draft and approval of final manuscript. A-KH: contributed in the design, revision of draft and approval of final manuscript. BH: contributed in the conception, planning, design, revision of draft and approval of final manuscript. JL: contributed in the conception, planning, design, revision of draft and approval of final manuscript. AlL: contributed in the conception, planning, design, revision of draft and approval of final manuscript. TWL: contributed in the design, revision of draft and approval of final manuscript. JEN: contributed in the conception, planning, design, revision of draft and approval of final manuscript. TM0: contributed in the design, revision of draft and approval of final manuscript. ElS: contributed in the conception, planning, design, revision of draft and approval of final manuscript. EiS: contributed in the conception, planning, design, analysis and interpretation of data, revision of draft and approval of final manuscript. JK-J: contributed in the conception, planning, design, acquisition of data, analysis and interpretation of data, first draft of article, revision of draft and approval of final manuscript.

Funding The authors have not declared a specific grant for this research from any funding agency in the public, commercial or not-for-profit sectors.

Map disclaimer The depiction of boundaries on this map does not imply the expression of any opinion whatsoever on the part of BMJ (or any member of its group) concerning the legal status of any country, territory, jurisdiction or area or of its authorities. This map is provided without any warranty of any kind, either express or implied.

Competing interests There are no competing interests to report for the submitted work. TM0 reports grants from Zoll Foundation and grants from Laerdal foundation, outside the submitted work. TWL reports paid and unpaid consultant work for Lærdal Medical and the Norwegian Air Ambulance Foundation outside the submitted work.

\section{Patient consent for publication Not required.}

Ethics approval This study was approved by Regional Ethical Committee (REK 60984) and data handling was done in accordance with recommendations from the local Data Protection Officer at Oslo University Hospital (20/01070).

Provenance and peer review Not commissioned; externally peer reviewed.

Data availability statement Data may be obtained from a third party and are not publicly available. The data that support the findings of this study are available from the Norwegian Cardiac Arrest Registry through an application to the Norwegian Institute of Public health. Restrictions apply to the availability of these data, as only aggregated data can be shared unless there is an approval from an ethical committee and the steering committee of the registry.

Open access This is an open access article distributed in accordance with the Creative Commons Attribution Non Commercial (CC BY-NC 4.0) license, which permits others to distribute, remix, adapt, build upon this work non-commercially, and license their derivative works on different terms, provided the original work is properly cited, appropriate credit is given, any changes made indicated, and the use is non-commercial. See: http://creativecommons.org/licenses/by-nc/4.0/.

\section{ORCID iD}

Ingvild Beathe Myrhaugen Tjelmeland http://orcid.org/0000-0003-0362-1008

\section{REFERENCES}

1 Meaney PA, Bobrow BJ, Mancini ME, et al. Cardiopulmonary resuscitation quality: [corrected] improving cardiac resuscitation outcomes both inside and outside the hospital: a consensus statement from the American Heart Association. Circulation 2013;128:417-35.

2 Hazinski MF, Nolan JP, Billi JE, et al. Part 1: Executive summary: 2010 international consensus on cardiopulmonary resuscitation and emergency cardiovascular care science with treatment recommendations. Circulation 2010;122:S250-75.

3 Global Resuscitation Alliance. Secondary global resuscitation alliance 2017. Available: https://www.globalresuscitationalliance.org [Accessed 21 Apr 2020].

4 Gräsner J-T, Wnent J, Herlitz J, et al. Survival after out-of-hospital cardiac arrest in Europe - Results of the EuReCa TWO study. Resuscitation 2020;148:218-26.

5 Beck B, Bray J, Cameron P, et al. Regional variation in the characteristics, incidence and outcomes of out-of-hospital cardiac arrest in Australia and New Zealand: results from the Aus-ROC Epistry. Resuscitation 2018;126:49-57.

6 Årsrapport HJ. Yearly report of 2017 from the Swedish cardiac arrest registry. 2018. Svenska Hjärt-Lungräddningsregistret, 2017.

7 Lindner TW, Søreide E, Nilsen OB, et al. Good outcome in every fourth resuscitation attempt is achievable--an Utstein template report from the Stavanger region. Resuscitation 2011;82:1508-13.

8 Nilsen JML, Haug B, Rasmussen JR, Clinic of Emergency Medicine, Universitry Hosp of North Norway, Tromsoe, Norway. Doubled survival from out-of-hospital cardiac arrest in a rural community in North-Norway following implementation of an aggressive chest pain protocol with early prehospital thrombolysis for STEMI. Circulation 2011.

9 Helse- og omsorgsdepartementet. Forskrift Om innsamling OG behandling AV helseopplysninger I Nasjonalt register over hjerte- OG karlidelser (personal health data filing system act. Oslo: Helse- og msorgsdepartementet, 2014. 
10 Perkins GD, Jacobs IG, Nadkarni VM, et al. Cardiac arrest and cardiopulmonary resuscitation outcome reports: update of the Utstein resuscitation registry templates for out-of-hospital cardiac arrest: a statement for healthcare professionals from a task force of the International liaison Committee on resuscitation (American heart association, European resuscitation Council, Australian and New Zealand Council on resuscitation, heart and stroke Foundation of Canada, InterAmerican heart Foundation, resuscitation Council of southern Africa, resuscitation Council of Asia); and the American heart association emergency cardiovascular care Committee and the Council on cardiopulmonary, critical care, perioperative and resuscitation. Resuscitation 2015;96:328-40.

11 R Foundation for Statistical Computing. A language and environment for statistical computing 2017.

12 Tjomsland N, Baskett P, Laerdal AS. Asmund S. Laerdal. Resuscitation 2002;53:115-9.
13 The Directorate of Health. Saving lives together. helsedirektoratet, 2018.

14 Maurer $\mathrm{H}$, Masterson S, Tjelmeland IB, et al. When is a bystander not a bystander any more? a European survey. Resuscitation 2019;136:78-84.

15 Beck B, Bray JE, Smith K, et al. Description of the ambulance services participating in the Aus-ROC Australian and New Zealand out-of-hospital cardiac arrest Epistry. Emerg Med Australas 2016;28:673-83.

16 Ringgren KB, Christensen HC, Schønau L. Dansk hjertestopregister 2018 Danish cardiac arrest registry, 2019.

17 Strömsöe A, Svensson L, Axelsson Å B, et al. Validity of reported data in the Swedish cardiac arrest register in selected parts in Sweden. Resuscitation 2013;84:952-6.

18 Becker LB, Smith DW, Rhodes KV. Incidence of cardiac arrest: a neglected factor in evaluating survival rates. Ann Emerg Med 1993;22:86-91. 